\title{
Effect of varying hypoxia reoxygenation times on autophagy of cardiomyocytes ${ }^{1}$
}

\author{
Zhao Hu', Hong-Yan Cai", Yun-Yan Luo'"', Jian-Ming Xiao'v, Lin Li", Tao Guo'v
}

'Master, Department of Cardiology, the First Affiliated Hospital of Kunming Medical University, Kunming, China. Conception and design of the study, acquisition and interpretation of data.

"PhD, Associate Professor, Department of Cardiology, the First Affiliated Hospital of Kunming Medical University, Kunming, China. Analysis and interpretation of data.

I"'Graduate student, Department of Cardiology, the First Affiliated Hospital of Kunming Medical University, Kunming, China. Acquisition and interpretation of data.

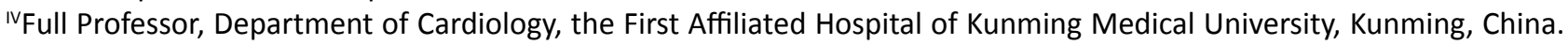
Critical revision.

\begin{abstract}
Purpose: To investigate the impact of different hypoxia reoxygenation (HR) times on autophagy of rat cardiomyocytes (H9C2).
\end{abstract}

Methods: Rat cardiomyocytes were randomly divided into normal control group (group A), hypoxia group (group B), 2 h HR group (group C), 12 h HR group (group D), and $24 \mathrm{~h}$ HR group (group E). LC3 II/LC3 I was determined via western blotting, and cell viabilities of cardiomyocytes were measured using methyl thiazolyl tetrazolium (MTT) assay.

Results: Cell viabilities in HR model groups were significantly lower than those of group A $(P<0.05)$. LC3 II/LC3 I levels in groups $B$ to $D$ were significantly higher than those of group $A$ $(P<0.05)$, and group $D$ showed the highest LC3 II/LC3 I levels. Cell viabilities in groups $B$ to $D$ were significantly lower than those of group $A(P<0.05)$, with group $D$ showing the lowest cell viabilities $(\mathrm{P}<0.05)$.

Conclusions: Hypoxia can induce autophagy in rat cardiomyocytes, which can be further activated by reoxygenation; most notable after $12 \mathrm{~h}$. Hypoxia-induced cell injury can be aggravated by reoxygenation. The lowest cell viability was observed at $12 \mathrm{~h}$ after reoxygenation; however, cell viability can be recovered after $24 \mathrm{~h}$.

Key words: Autophagy. Myocytes, Cardiac. Hypoxia. Rats. 


\section{Introduction}

Autophagy is a process in which bilayer vesicles (autophagosomes) are generated within the cell. Autophagosomes then hydrolyze damaged cellular components, such as protein aggregates, dysfunctional mitochondria, or damaged endoplasmic reticulum. Autophagy can be activated by nutrient deficiency, hypoxia, ischemia-reperfusion, or other factors ${ }^{1}$. Autophagy can be classified using the following two methods. First, autophagy can be classified into macroautophagy, microautophagy, or chaperon-mediated autophagy (CMA) based on the process by which intracellular substrates are transported into the lysosomal cavity ${ }^{2}$. Of these, macroautophagy is the most widely studied. Second, autophagy can be classified into selective or nonselective autophagy according to selectivity towards target substrates $^{3}$. Nonselective autophagy refers to the transportation and degradation of random cytoplasmic organelles by lysosomes. Selective autophagy exhibits specificity towards the target substrates ${ }^{4}$ and can be further classified into the following subtypes according to the selectivity of substrate proteins: mitophagy ${ }^{5}$, nucleophagy $^{6}$, lipophagy ${ }^{7}$, ribophagy ${ }^{8}$, and xenophagy ${ }^{9}$. Additional types of selective autophagy are expected to be discovered in future studies. Autophagy is associated with various diseases, such as cancer, nervous system diseases, and cardiovascular diseases. Thus, autophagy regulation has become a therapeutic target for the treatment of these diseases ${ }^{10}$. The autophagy process must be terminated once adverse physiological states have been overcome. Otherwise, non-specific degradation of cytoplasmic components can lead to irreversible cell damage. Therefore, autophagy is a dynamic process that requires careful and systematic investigation. In the present study, we used $\mathrm{H} 9 \mathrm{C} 2$ cells to detect the changes in expression of the autophagyrelated protein LC3 under different hypoxia reoxygenation (HR) periods and to observe the impact of autophagy-induced changes on the cell viability of cardiomyocytes. We further explored changes in autophagy after exposure to hypoxia to elucidate mechanisms with the goal of achieving fine autophagy control.

\section{Methods}

\section{Cell culture and treatment}

The H9C2 cells were purchased from Xiangya School of Medicine (Changsha, Hunan, China) and cultured in high-glucose Dulbecco's minimum essential medium (DMEM) containing $10 \%$ fetal bovine serum (FBS) and $1 \%$ double antibodies (Hyclone, USA). The medium was replaced once daily, and cells were passaged every 3 to 4 days.

\section{Treatment groups and culture conditions}

For normal culture, cells were grown in DMEM containing $10 \% \mathrm{FBS}$ at $37^{\circ} \mathrm{C}$ and $5 \%$ $\mathrm{CO}_{2}$. For hypoxic culture, cells were cultured in serum-free low-glucose DMEM at $37{ }^{\circ} \mathrm{C}$, $93 \% \mathrm{~N}_{2}, 5 \% \mathrm{CO}_{2}$, and $2 \% \mathrm{O}_{2}$. For reoxygenated culture, cells were cultured under normal conditions following hypoxic culture.

HR model groups: H9C2 cells were grown until they covered approximately $90 \%$ of the bottom of the culture flask. Cells were then randomly divided into two groups $(n=3)$ and cultured in DMEM based on the following treatments: (1) normal control group (group A) was cultured under normal conditions; (2) HR model group (B group) was cultured for 2 $h$ under normal conditions, followed by $2 \mathrm{~h}$ of hypoxic culture.

The HR model groups were subjected to the following treatments: (1) normal control group (group A) was cultured under normal conditions; (2) hypoxia group (group B) was cultured for $2 \mathrm{~h}$ under hypoxic conditions; (3) $2 \mathrm{~h} \mathrm{HR}$ group (group C) was cultured under 
normal conditions for $2 \mathrm{~h}$, followed by $2 \mathrm{~h}$ of hypoxic culture; (4) 12 h HR group (group D) was cultured under normal conditions for 12 h, followed by $2 \mathrm{~h}$ of hypoxic culture; (5) $24 \mathrm{~h}$ HR group (group $\mathrm{E}$ ) was cultured under normal conditions for $24 \mathrm{~h}$, followed by $2 \mathrm{~h}$ of hypoxic culture.

\section{Methyl thiazolyl tetrazolium (MTT) assay}

Cells were inoculated into 96-well culture plates. After the corresponding treatments, the MTT solution (Biosharp, Hefei, China) was added to the cardiomyocytes, followed by $4 \mathrm{~h}$ of culture and addition of the triple solution for overnight culture at a constant-temperature incubator. The next day, the optical density $(A)$ in each well was measured thrice at $570 / 630 \mathrm{~nm}$ using a microplate reader. The mean value was used for calculating cell viability using the following formula: cell viability = A value of each well / average $A$ value of the control group $\times 100 \%$.

\section{Western blot analysis}

Total proteins of cardiomyocytes were extracted and quantified using the BCA method (Beyotime Biotechnology Co. Ltd., Shanghai, China). The loading volume of each sample was calculated based on the protein concentrations; samples were mixed with the loading buffer of sodium dodecyl sulfate (SDS) gel, and then denatured for $5 \mathrm{~min}$ in a dry bath at $95^{\circ} \mathrm{C}$. Samples were then stored at $-20^{\circ} \mathrm{C}$. Samples were then subjected to sodium dodecyl sulfate polyacrylamide gel electrophoresis (SDS-PAGE) using $15 \%$ separation gel and $5 \%$ compression gel. Electrophoresis was performed under the following conditions: $30 \mathrm{~min}$ in the compression gel at $80 \mathrm{~V}$, followed by $90 \mathrm{~min}$ in the separation gel at $110 \mathrm{~V}$. After separation, proteins were transferred onto a PVDF membrane (35 min at $15 \mathrm{~V}$ ) and subsequently labeled with standard proteins. The membrane was then cut according to different protein molecular weights and rinsed four times with TBST in a small box (5 min per wash). The PVDF membrane was blocked using $5 \%$ skim milk in TBST for $1.5 \mathrm{~h}$ at room temperature $\left(18-22^{\circ} \mathrm{C}\right)$ and rinsed four times with TBST for $5 \mathrm{~min}$ each wash. Afterwards, the primary LC3A/B antibody (Cell Signaling Technology, batch no. 4108, 1:1000 dilution) and rabbit GAPDH (14C10) monoclonal antibody as internal control (Cell Signaling Technology, batch no. 4118, 1:2000 dilution) were added, followed by overnight incubation with shaking. After removing the primary antibodies, the film was rinsed four times with TBST for $5 \mathrm{~min}$ each, added with secondary antibodies (R-1:10000 LC3 and R-1:40000 GAPDH), and incubated for $90 \mathrm{~min}$ with shaking at room temperature $\left(18-22{ }^{\circ} \mathrm{C}\right)$. After rinsing four times with TBST for 5 min each wash, the film was developed. The average optical density of each strip was measured using a chemiluminescence highsensitivity gel imaging and analysis system to determine the relative expression levels of the target protein.

\section{Statistical analysis}

All data were expressed as ( $\bar{x} \pm \mathrm{s})$. Data analyses were performed using SPSS17.0 software package. The independent-sample t-test was used to perform intergroup comparisons, while multi-group differences were analyzed using one-way ANOVA. PostANOVA LSD test was used for intergroup pairwise comparisons. Excel was used for generating graphs.

\section{- Results}

\section{Validation of the HR injury model}

Cardiomyocytes in groups $\mathrm{B}$ and $\mathrm{A}$ had cell viabilities of $58.97 \pm 1.20 \%$ and $92.84 \pm 1.49 \%$, 
respectively, demonstrating significantly lower cell viability in the HR model group compared to the normal control group $(P<0.05)$.

\section{LC3 expression}

Protein expression of LC3 in the cardiomyocytes was detected by western blotting. During autophagy, the cytoplasmic LC3 (LC3 I) is cleaved into a small polypeptide and converted to membrane LC3 (autophagosomal LC3 II). Thus, the LC3 II/LC3 I ratio can be used to evaluate the LC3 expression. Groups B, C, $D$, and $E$ had significantly higher LC3 II/LC3 I ratios than group $A(P<0.05)$, indicating that hypoxia and HR can induce LC3 upregulation. In addition, the LC3 II/LC3 I ratios of groups C and $D$, especially group $D$, were significantly higher than that of group $B(P<0.05)$. Groups $B$ and $E$ showed no significant differences in LC3 II/LC3 I ratio (Table 1, Figure 1).

Table 1 - Impact of different HR time on expression of LC3 protein in cardiomyocytes ( $\bar{x} \pm \mathrm{s})$.

\begin{tabular}{ccc} 
Groups & $\mathbf{n}$ & LC3 II/LC3 I (Ratio of Control) \\
\hline A & 3 & $1.0000 \pm 0.0000$ \\
B & 3 & $4.3400 \pm 0.20000^{*}$ \\
C & 3 & $5.0833 \pm 0.24664^{* \#}$ \\
D & 3 & $6.7367 \pm 0.38109^{* \Delta}$ \\
E & 3 & $3.9900 \pm 0.05292^{*}$ \\
\hline
\end{tabular}

Note: ${ }^{*} P<0.05$ vs. group $A,{ }^{\sharp} P<0.05$ vs. group $B, \triangle P<0.05$ vs. group $C$.

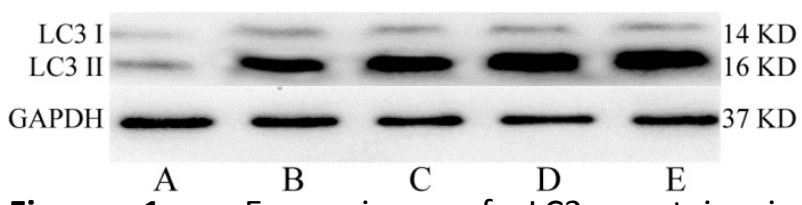

Figure 1 - Expressions of LC3 protein in cardiomyocytes.

\section{Viability of cardiomyocytes}

The cell viability in each group was measured using MTT assay. Cell viabilities of groups B, C, D, and E were significantly lower than that of group $A(P<0.05)$, indicating that hypoxia and $H R$ can reduce cell viability of cardiomyocytes. Cell viabilities of groups $\mathrm{C}$ and $D$, especially group $D$, were significantly lower than that of group $B(P<0.05)$. Cell viability of group $E$ was higher than that of group $B$ $(\mathrm{P}<0.05)$ (Table 2).

Table 2 - Impact of different HR time on viability of cardiomyocytes ( $\bar{x} \pm \mathrm{s})$.

\begin{tabular}{ccc} 
Groups & $\mathbf{n}$ & Cell viability (\%) \\
\hline A & 3 & $94.07 \pm 1.57$ \\
B & 3 & $66.04 \pm 1.61^{*}$ \\
C & 3 & $62.29 \pm 1.99^{* \#}$ \\
D & 3 & $51.05 \pm 1.94^{*}$ \\
E & 3 & $72.57 \pm 2.29^{* \Delta}$ \\
\hline
\end{tabular}

Note: ${ }^{*} P<0.05$ vs. group $A,{ }^{\#} P<0.05$ vs. group $B, \triangle{ }^{\Delta} P<0.05$ vs. group $C,{ }^{\Delta} P<0.05$ vs. group $B$.

\section{Discussion}

Myocardial IR injury is generally believed to be caused by multiple factors. The pathophysiology of myocardial IR injury is mediated by apoptosis, intracellular oxygen free radical formation, $\mathrm{Ca}^{2+}$ overload, neutrophil activation, and release of inflammatory factors. An increasing number of studies have demonstrated the crucial role of autophagy in myocardial IR injury. However, autophagy is a complex process that has diverse biological functions and physiological roles in different cell types, cellular states, and developmental stages.

Establishment of HR injury model: $\mathrm{H} 9 \mathrm{C} 2$ cells were obtained from rat embryonic cardiomyocytes. H9C2 cells cannot pulsate but are still widely used in cytological and pharmacological research because they exhibit the physiological and biochemical characteristics of primary cardiomyocytes ${ }^{11}$ and can be subcultured. In vitro culture of cardiomyocytes 
can eliminate interference from systemic nerves and humoral factors and thus facilitate the investigation of the effects of relevant factors on the pathophysiology of individual cells and their underlying mechanisms. In evaluating myocardial ischemia-reperfusion injury, the viability of cardiomyocytes can be used as the primary index to assess damage to cardiomyocytes in vitro. In the present study, cardiomyocytes were subjected to low-glucose hypoxia (2\%) to simulate the ischemic and hypoxic conditions, after which glucose and oxygen supplies were restored to simulate the reperfusion. Meanwhile, we induced changes in the interstitial fluid during ischemia/ reperfusion (hypoxia, high potassium, lactic acid accumulation, acidosis, sugar depletion, and return to normal after reperfusion) to simulate ischemia-reperfusion injury. Literature indicated that oxygen concentration during hypoxic culture should range from $0 \%$ to $5 \%^{12,13}$. In this experiment, we selected $2 \%$ $\mathrm{O}_{2}$, which can effectively achieve the desired hypoxic culture conditions and simulate myocardial ischemic injury. Results showed that the viability of cardiomyocytes in the model group was significantly lower than that of the normal control group $(P<0.05)$, demonstrating the effectiveness and feasibility of the in vitro HR injury model established using the above method.

Effect of different HR times on autophagy expression and cell viability: This study investigated the changes in autophagy and cell viabilities of cardiomyocytes subjected to varying $H R$ periods. Upon receiving the autophagy-inducing signal, cells first form an electron microscopically observable membrane structure in the cytoplasm that extends within the cell, such that small membrane structures are formed. The membrane structure then continues to expand and eventually ingests the mitochondria, endoplasmic reticulum, or other organelles in the cytoplasm to form autophagosomes. The autophagosomes subsequently fuse with lysosomes to form the autophagic lysosomes. In turn, autophagic lysosomes degrade the autophagic intimal membrane and contents and generate amino acids, fatty acids, or other products in the cytoplasm, which are subsequently reused by cells. LC3 is a mammalian homolog of the yeast autophagic gene (Apg7/Apg8). LC3 accumulates during autophagy and is the only protein expressed on the autophagosome membrane and has consequently been widely used as an autophagic marker ${ }^{14}$. LC3 is dispersed in the cytoplasm under normal conditions but becomes translocated to the autophagic membrane during autophagy ${ }^{15}$. Western blotting can be used to detect the cytoplasmic LC3 (LC3 I) and membrane LC3 (LC3 II) levels and calculate the LC3 II/LC3 I ratio, which can then be used to evaluate the expression of LC3 protein.

Relationship between $\mathrm{HR}$ times and autophagy: LC3 protein expression among the treatment groups is given the following decreasing order: groups $D, C, B, E$, and $A$. LC3 expression in group $B$ was significantly higher than that in group $A(P<0.05)$, and $L C 3$ expression in group $C$ was significantly higher than that in group $B(P<0.05)$. These results indicate that hypoxia can induce autophagy in cardiomyocytes and that reoxygenation can further stimulate autophagy, consistent with the previous report by Matsui et al. ${ }^{16}$. Throughout the three different HR stages, LC3 expression started to increase at $2 \mathrm{~h}$ and reached peak expression at $12 \mathrm{~h}(\mathrm{P}<0.05)$. However, LC3 expression at $24 \mathrm{~h}$ was lower than that at $12 \mathrm{~h}(\mathrm{P}<0.05)$ and follows a decreasing trend, but there was no statistically significant difference in LC3 expression between these two groups $(P<0.05)$.

The above results suggest that the autophagy process is different in hypoxia and reoxygenation stages. Previous studies 
provided evidence that myocardial autophagy is activated in the hypoxic phase as a result of depletion in available nutrient and energy supplies, which leads to reduction of intracellular ATP levels. In turn, this activates the adenosine monophosphate-activated kinase (AMPK) and simultaneously inhibits the cellular amino acid and ATP receptor mammalian target of rapamycin (mTOR) ${ }^{17}$, thereby enhancing autophagy. Reoxygenation restores the nutrient and energy supplies, so that AMPK will be inactivated and can no longer inhibit mTOR. Therefore, the mechanism underlying considerable amplification of autophagic activation has no direct relationship with AMPK activation and mTOR inhibition, and is only related to the upregulation of the autophagic gene Beclin 1. As a result, Beclin1 binds to class III phosphatidylinositol 3 kinase (class III PI3K) to form the Beclin1-class III PI3K complex, which further upregulates autophagy ${ }^{18}$. Varying degrees of autophagy at different HR times may be caused by different intracellular Beclin1 levels. However, further experiments must be conducted to validate these results.

Relationship between HR time and viability of cardiomyocytes: Results of this study showed that the different treatment groups had cell viabilities in the following decreasing order: group $A>$ group $E>$ group $B>$ group $C>$ group $D$. Cell viability in group $B$ was significantly lower than that in group $A(P<0.05)$, indicating that hypoxia caused cardiomyocyte injury, which was reduced during reoxygenation $(P<0.05)$. Group D showed the lowest cell viabilities in all reoxygenation periods $(P<0.05)$. Cell viability was higher in group $E$ than in group $B$ but remained lower than that in group $A(P<0.05)$, indicating that cardiomyocyte injury was induced by hypoxia but was reduced during reoxygenation. Studies also have demonstrated that long-term exposure to continuous hypoxia led to significantly greater injury in $\mathrm{H} 9 \mathrm{C} 2$ cells compared to reoxygenation ${ }^{19}$. Thus, in-time reperfusion after ischemia is necessary for cardiomyocytes, and effective intervention is crucial to reduce ischemia-reperfusion injury.

The relationship between autophagy and cell viability: Viability of myocardial cells was found to decrease with autophagy upregulation in groups $B, C$, and D, indicating that increasing autophagy activation causes more severe cell injury. Group D showed the highest degree of autophagy and lowest viability of myocardial cells. Groups E and B showed no difference in autophagy; however, cell viability was higher in group $B(P<0.05)$. Studies have demonstrated that autophagy activation in mildly ischemic cardiomyocytes can reduce cell death ${ }^{20}$. However, autophagy can be significantly activated during the reperfusion period, which promotes cell death $^{21}$. Consistent with the abovementioned findings, results of our study showed that autophagy was upregulated in groups $C$ and $D$ and was accompanied by decreased cell viabilities. Similar to the results for group $B$, autophagy was activated in group $E$, but cell viability was higher in group $B(P<0.05)$. These observations can be attributed to the long reoxygenation time ( $24 \mathrm{~h})$, during which nutrient and energy supplies are restored, allowing cardiomyocytes to restore growth.

\section{Conclusions}

Hypoxia can induce autophagy in rat cardiomyocytes, which can be further activated by reoxygenation; most notable after $12 \mathrm{~h}$. Hypoxia-induced cell injury can be aggravated by reoxygenation. The lowest cell viability was observed at $12 \mathrm{~h}$ after reoxygenation; however, cell viability can be recovered after $24 \mathrm{~h}$.

\section{References}

1. Dong $Y$, Undyala VV, Gottlieb RA, Mentzer RM Jr, Przyklenk K. Autophagy: definition, molecular machinery, and potential role in 
myocardial ischemia-reperfusion injury. J Cardiovasc Pharmacol Ther. 2010;15(3):22030. doi: $10.1177 / 1074248410370327$.

2. Nakai A, Yamaguchi O, Takeda T, Higuchi Y, Hikoso S, Taniike M, Omiya S, Mizote I, Matsumura Y, Asahi M, Nishida K, Hori M, Mizushima N, Otsu K. The role of autophagy in cardiomyocytes in the basal state and in response to hemodynamic stress. Nat Med. 2007;13(5):619-24. doi: 10.1038/nm1574.

3. Stolz A, Ernst A, Dikic I. Cargo recognition and trafficking in selective autophagy. Nat Cell Biol. 2014;16(6):495-501. doi: 10.1038/ ncb2979.

4. Okamoto K. Organellophagy: etimilating cellular building blocks via selective autophagy. J Cell Biol. 2014;205(4):435-45. doi: $10.1083 /$ jcb.201402054.

5. Lemasters JJ. Variants of mitochondial autophagy: Types 1 and 2 mitophagy and micromitophagy (Type3). Redox Biol. 2014;2:749-54. doi: 10.1016/j. redox.2014.06.004. eCollection 2014.

6. Mijaljica D, Devenish RJ. Nucleophagy at a glance. J Cell Sci. 2013;126(Pt 19):4325-30. doi: $10.1242 /$ jcs.133090.

7. Khaldoun SA, Emond-Boisjoly MA, Chateau $D$, Carrière V, Lacasa M, Rousset M, Demignot $\mathrm{S}$, Morel E. Autophagosomes contribute to intracellular lipid distribution in enterocytes. Mol Biol Cell. 2014;25(1):118-32. doi: 10.1091/mbc.E13-06-0324.

8. Cebollero E, Reggiori F, Kraft C. Reticulophagy and ribophagy: regulated degradation of protein production factories. Int J Cell Biol. 2012;2012:182834. doi: 10.1155/2012/182834.

9. Knodler LA, Celli J. Eating the strangers within: host control of intracellular bacteria via xenophagy. Cell Microbiol. 2011;13(9):1319-27. doi: 10.1111/j.14625822.2011.01632.x.

10.Schneider JL, Cuervo AM. Autophagy and human disease: emerging themes. Curr Opin Genet Dev. 2014;26:16-23. doi: 10.1016/j. gde.2014.04.003.

11. Hescheler J, Meyer R, Plant S, Krautwurst D, Rosenthal W, Schultz G. Morphological, biochemical, and electrophysiological characterization of a clonal cell ( $\mathrm{H} 9 \mathrm{c} 2)$ line from rat heart. Circ Res. 1991;69(6):147686. doi: 10.1161/01.RES.69.6.1476.

12.Zhang ZL, Fan Y, Liu ML. Ginsenoside Rg1 inhibits autophagy in H9c2 cardiomyocytes exposed to hypoxia/reoxygenation. Mol Cell Biochem. 2012;365(1-2):243-50. doi: 10.1007/s11010-012-1265-3.

13.Lee S, Kim K, Kim YH, Chung MH, Kang I, $\mathrm{Ha}$ J, Choe W. Preventive role of propofol in hypoxia/reoxygenation-induced apoptotic H9c2 rat cardiac myoblast cell death. Mol Med Rep. 2011;4(2):351-6. doi: 10.3892/ mmr.2011.432.

14. Kabak YB, Sozmen M, Yarim $M$, Guvenc T, Karayigit MO, Gulbahar MY. Immunohistochemical detection of autophagy-related microtubuleassociated protein 1 light chain 3 (LC3) in the cerebellums of dogs naturally infected with canine distemper virus. Biotech Histochem. 2015;90(8):601-7. doi: 10.3109/10520295.2015.1064999.

15.Tanida I, Ueno T, Kominami E. LC3 and autophagy. Methods Mol Biol. 2008;445:7788. doi: 10.1007/978-1-59745-157-4 4.

16. Matsui $Y$, Takagi $H, Q u$ X, Abdellatif $M$, Sakoda $H$, Asano T, Levine B, Sadoshima $J$. Distinct roles of autophagy in the heart during ischemia and reperfusion: roles of AMP-activated protein kinase and Beclin 1 in mediating autophagy. Circ Res. 2007;100(6):914-22. doi: 10.1161/01. RES.0000261924.76669.36

17.Alers S, Löffler AS, Wesselborg S, Stork B. Role of AMPK-mTOR-Ulk1/2 in the regulation of autophagy: cross talk, shortcuts, and feedbacks. Mol Cell Biol. 2012;32(1):2-11. doi: 10.1128/MCB.06159-11.

18. Castino R, Bellio N, Follo C, Murphy D, Isidoro C. Inhibition of PI3K class IIIdependent autophagy prevents apoptosis and necrosis by oxidative stress in dopaminergic neuroblastoma cells. Toxicol Sci. 2010;117(1):152-62. doi: 10.1093/ toxsci/kfq170.

19.Bonavita F, Stefanelli C, Giordano E, Columbaro $M$, Facchini $A$, Bonafè $F$, Caldarera CM, Guarnieri C. H9c2 cardiac myoblasts undergo apoptosis in a model of ischemia consisting of serum deprivation and hypoxia: inhibition by PMA. FEBS Lett. 2003;536(1-3):85-91. doi: 10.1016/S00145793(03)00029-2.

20.Sciarretta S, Hariharan N, Monden Y, Zablocki $D$, Sadoshima J. Is autophagy in response to ischemia and reperfusion protective or 
detrimental for the heart? Pediatr Cardiol. 2011;32(3):275-81. doi: 10.1007/s00246010-9855-x.

21.Nishida K, Kyoi S, Yamaguchi O, Sadoshima
J, Otsu K. The role of autophagy in the heart. Cell Death Differ. 2009;16(1):31-8. doi: 10.1038/cdd.2008.163.

\section{Correspondence:}

Tao Guo

Department of Cardiology

The First Affiliated Hospital of Kunming Medical University

No. 295 Xichang Road

Kunming 650032 China

Phone: +8687165377618

doctaoguo@163.com

Received: Nov 07, 2017

Review: Jan 08, 2018

Accepted: Feb 06, 2018
Conflict of interest: none

Financial sources: National Natural Science Foundation of China (No.81560072, No.81760067), Yunnan Provincial Science and Technology Department (No.2013FB128, 2014FB037) and Yunnan Provincial Medical Science Leader Training Project (D-201622)

${ }^{1}$ Research performed at Center of Pharmacy, Department of Pharmacy, Kunming Medical University, China. 